This is a preprint of the article: Foerster, R. M. \& Schneider, W. X. (2018). Involuntary top-down control by search-irrelevant features: Visual working memory biases attention in an object-based manner. Cognition, 172,

37-45. doi: 10.1016/j.cognition.2017.12.002. The article has been published in its final form at https://doi.org/10.1016/j.cognition.2017.12.002. (c) 2018. This manuscript version is made available under the CC-BY-NC-ND 4.0 license http://creativecommons.org/licenses/by-nc-nd/4.0/

\title{
Involuntary top-down control by search-irrelevant features: Visual working memory biases attention in an object-based manner
}

\author{
Rebecca M. Foerster (rebecca.foerster@uni-bielefeld.de) ${ }^{1,2}$, Werner X. Schneider ${ }^{1,2}$ \\ ${ }^{1}$ Neuro-cognitive Psychology, Bielefeld University \\ ${ }^{2 ‘}$ Cognitive Interaction Technology’ Cluster of Excellence CITEC, Bielefeld University
}

Running head: Involuntary top-down control by VWM templates

Correspondence: Rebecca M. Foerster, Neuro-cognitive Psychology, Department of Psychology, Bielefeld University, P.O. Box 100131, D-33501 Bielefeld, Germany, +49521/106-4503, rebecca.foerster@uni-bielefeld.de 
Abstract: Many everyday tasks involve successive visual-search episodes with changing targets. Converging evidence suggests that these targets are retained in visual working memory (VWM) and bias attention from there. It is unknown whether all or only searchrelevant features of a VWM template bias attention during search. Bias signals might be configured exclusively to task-relevant features so that only search-relevant features bias attention. Alternatively, VWM might maintain objects in the form of bound features. Then, all template features will bias attention in an object-based manner, so that biasing effects are ranked by feature relevance. Here, we investigated whether search-irrelevant VWM template features bias attention. Participants had to saccade to a target opposite a distractor. A colored cue depicted the target prior to each search trial. The target was predefined only by its identity, while its color was irrelevant. When target and cue matched not only in identity (search-relevant) but also in color (search-irrelevant), saccades went more often and faster directly to the target than without any color match (Experiment 1). When introducing a cuedistractor color match (Experiment 2), direct target saccades were most likely when target and cue matched in the search-irrelevant color and least likely in case of a cue-distractor color match. When cue and target were never colored the same (Experiment 3), cue-colored distractors still captured the eyes more often than different-colored distractors despite color being search-irrelevant. As participants were informed about the misleading color, the result argues against a strategical and voluntary usage of color. Instead, search-irrelevant features biased attention obligatorily arguing for involuntary top-down control by object-based VWM templates.

Keywords: object-based attention, visual search template, oculomotor capture, visual working memory, involuntary top-down control 


\section{Introduction}

Many everyday tasks involve successive visual-search episodes with changing targets. Before writing a note you have to look not only for a pencil, but also for a piece of paper in your vicinity. Surprisingly, mechanisms of visual search have mostly been investigated by means of experiments with constant search targets throughout a series of search trials (Treisman \& Gelade, 1980; Wolfe, 1994, 2010; Zelinsky, 2012). In this case, due to repetition of the same search target in each trial, a representation of relevant features of the target object can be recoded and retained in long-term memory (LTM, Woodman, Carlisle, \& Reinhart, 2013). If the target changes from one search episode to the next, as is the case in most everyday tasks, time might not suffice to recode a representation of the search target into an LTM template. Instead, a short-term memory (STM) also called working memory (WM) representation of the search target should be formed after target specification and maintained until the target is found (Woodman et al., 2013). This working memory representation of the search target is often called search template (e.g., Olivers, Peters, Houtkamp, \& Roelfsema, 2011).

Experimental evidence differentiates between STM/WM-based search for trial-wise varying targets and LTM-based search for constant targets over trials. Firstly, searches for changing targets are less efficient than searches for a constant target (Bravo \& Nakayama, 1992; Wolfe, Horowitz, Kenner, Hyle, \& Vasan, 2004). Second, there is evidence from human and non-human primate electrophysiology that STM and LTM representations of a search object differ. Woodman et al. (2013) measured contralateral-delay activity (CDA) while humans performed visual searches. The CDA is a relative negativity at parietal, occipital, and temporal electrodes that is supposed to indicate maintenance of visual representations in WM (Vogel \& Machizawa, 2004). Importantly, CDA activity stayed high throughout a series of searches with changing targets, while it decreased over the course of 
repeated searches for a constant target. Furthermore, in a monkey study by Rossi et al. (2001), constant target searches were not affected by prefrontal lesions, while changing target searches were impaired. Thus, WM templates for changing search targets and LTM templates for constant search targets are retained differently in the primate brain (Woodman et al., 2013).

How might a visual search target be retained in WM? A major viewpoint of visual working memory (VWM) stresses (D’Esposito \& Postle, 2015) that transiently available visual information needs to be kept active over a short period of time until it is used for a subsequent action. VWM can be considered as a capacity-limited visual memory system with short-term retention and manipulation functions (the visuo-spatial sketchpad in Baddeley, 1986; Baddeley \& Hitch, 1974). Its capacity seems to be limited to three to four items (Cowan, 2000; Fukuda, Awh, \& Vogel, 2010; Luck \& Vogel, 1997, 2013, but see also Ma, Husain, \& Bays, 2014). Importantly, the VWM capacity is limited more by the number of objects rather than by the number of visual features (Luck \& Vogel, 1997; Vogel, Woodman, \& Luck, 2001; but see also Alvarez \& Cavanagh, 2004; Hardman \& Cowan, 2015; Palmer, Boston, \& Moore, 2015). Based on these findings, some theories assume that bound features of an object rather than separated features are represented in VWM (Luck \& Vogel, 1997, 2013). A similar assumption has been raised by theories that emphasize the binding function of attention (Kahneman, Treisman, \& Gibbs, 1992) and theories that view visual attention and working memory in the service of action control (Schneider, 1995, 2013) and advanced cognitive operations (Oberauer, 2009).

Given these considerations on the nature of VWM, for visual search tasks with changing targets (e.g., trial-wise), a template of a search target could be retained in VWM as a visual object with integrated features. Alternatively, the template in VWM could contain only search-relevant features of the search target. In the former case, that is, if an object with 
integrated features is stored as search template in VWM, then not only task-relevant but also task-irrelevant features might exert an attentional biasing effect. This consideration seems plausible if VWM objects are conceptualized as bidirectional loops between visual feature maps and a VWM map with a limited number (about 4) of object pointers (Bundesen, Habekost, \& Kyllingsbaek, 2005). Feedback connections from the VWM map could mediate the attentional bias effect in the visual feature maps. In this case, all features of a VWM template are likely kept active through these reverberating loops and should bias attentional selection in an object-based manner. Consequently, even completely irrelevant features of an active VWM representation should bias attention via involuntary top-down control signals such as a bias by color of a VWM template when searching for a template-matching shape. So far, this question has not been studied experimentally, that is, whether all or only relevant features of a VWM template bias attention during the corresponding visual search with trialwise changing targets.

In the present study, we demonstrate in three experiments that a search-irrelevant feature, here color, of a VWM template biases attentional selection, arguing for involuntary object-based top-down control by irrelevant features. In all experiments, a search trial started with a colored cue depicting the search object at fixation. Afterwards, two colored objects appeared left and right from fixation. Participants had to saccade to the search object according to its identity and irrespective of its color. Overt saccadic eye movements were used as proxies for covert visual attention as it is known that a covert shift of attention obligatorily precedes each saccade (Deubel \& Schneider, 1996). In a first experiment, the target object appeared in the same color as the search cue in half of the trials. Although object identity and not color defined the target, first saccades went more often and faster towards the target if it appeared in the task-irrelevant cue's color rather than in any other color. As the distractor did never appear in the cue color, color might have been strategically used for 
search. In a second experiment, we therefore included a cue-distractor color-match condition. Cue-colored targets and distractors captured the eyes when they were accompanied by different-colored counterparts. Still, participants might have used the color voluntarily, as color was equally often valid and invalid. Therefore, in a third experiment, the target never appeared in the irrelevant cue color, while the distractor matched the cue color in half the trials. Furthermore, participants were informed that the cue color was never informative and even misleading in fifty percent of the trials. Although, it would have been strategically beneficial to ignore any cue-colored object in this case, first saccades landed still more often on a cue-colored than on a different-colored distractor. The results of these three experiments strongly support the view that VWM-search templates work obligatorily in an object-based manner during visual search tasks with trial-wise changing targets. All features of a VWM template, including the irrelevant features, seem to bias attentional selection due to an involuntary top-down regulation. Task knowledge cannot be used to eliminate the attentional and oculomotor capture from search-irrelevant template features completely.

\section{Experiment 1}

In Experiment 1, we investigated whether the search-irrelevant color feature of a trialwise varying target biases covert attention during visual search. Participants had to saccade to a target object in a search display. A search cue indicated the identity of the target object prior to each trial. Therefore, the search cue, that is, the target identity varied from trial to trial. The cue object and the search objects were always colored. However, color was not relevant for visual search because the target was defined only by its identity which could be extracted based on its shape only. We hypothesized that VWM templates work in an objectbased manner to bias attentional selection. Consequently, the search-irrelevant cue color should bias attention allocation and thus saccadic selection during the visual-search task. 


\subsection{Methods}

\subsubsection{Participants}

Eight students from Bielefeld University, Germany (5 males, 3 females; 26 years on average), participated in the experiment after having provided written informed consent. We chose a relatively small sample size of eight participants in all our experiments because we expected effects as large as found in earlier studies investigating how VWM influences visual selection in dual-task paradigms (e.g., ten participants in Soto, Heinke, Humphreys, \& Blanco, 2005; Soto, Humphreys, \& Heinke, 2006). A Cohen's $d_{z}$ of 2, a power of 0.99, and a chance level of 0.05 returns a needed sample size of six participants. Thus, our sample size of eight is similar to what a power analysis would have returned. All participants were naïve with respect to the study’s purpose, reported normal or corrected-to-normal visual acuity, and were paid for participation. All three studies were approved by the Committee for Ethics at the Department of Psychology, Bielefeld University.

\subsubsection{Apparatus and stimuli}

Stimuli were displayed on a 100-Hz and 19-in color monitor (View Sonic Graphics series G90fB, Brea, CA) with a spatial resolution of 1,024 x 768 pixels extending 36 x $27 \mathrm{~cm}$ using a Dell Precision T3600 with an NVIDIA GeForce GTX 970 graphics card. Participants’ right gaze position was recorded at 1,000 Hz by an SR Research EyeLink 1000 desktop eye tracker. A chin-and-forehead rest stabilized participants' heads at a viewing distance of 71 cm. The SR Research Experiment Builder software was used to control the experimental procedure. Luminance and color of the used stimuli were measured at screen center in CIE Lxy coordinates using an X-Rite i1 Pro spectrophotometer. Stimuli were displayed on a grey background (RGB 245,245,245; $\mathrm{L}=100.1 \mathrm{~cd} / \mathrm{m}^{2}, \mathrm{x}=0.3, \mathrm{y}=0.3$ ). A black plus (RGB 0,0,0; 
$\left.\mathrm{L}=0.1 \mathrm{~cd} / \mathrm{m}^{2}, \mathrm{x}=0.3, \mathrm{y}=0.3\right)$ subtending 0.45 degrees of visual angle $\left({ }^{\circ} \mathrm{V} . \mathrm{a}.\right)$ served as a central-fixation marker. Search stimuli consisted of four completely saturated colored realworld object shapes: a baby bottle, a bucket, a can, and a pot (Figure 1). The stimuli were modified objects from the object databank originally used in Konkle, Brady, Alvarez, and Oliva (2010), obtained from http://cvcl.mit.edu/MM/objectCategories.html. Matlab R2013b was used to adjust stimuli sizes and to substitute each object's texture with a single color. Search stimuli appeared in four different colors: green (RGB 0,200,0;60.7cd/m², $\mathrm{x}=0.3, \mathrm{y}=$ 0.6), blue (RGB 0,0,200; 9.9cd $/ \mathrm{m}^{2}, \mathrm{x}=0.2, \mathrm{y}=0.1$ ), yellow (RGB 200,200,0; $72.5 \mathrm{~cd} / \mathrm{m}^{2}, \mathrm{x}=$ $0.4, y=0.5$ ), and red (RGB 200,0,0;20.0cd $\left./ \mathrm{m}^{2}, x=0.6, y=0.3\right)$. The size of all search stimuli was 49 x 49 pixels, extending $1.39^{\circ}$ v.a. in foveal vision. Search cues were presented in the center. Search target and distractor were located $5 \cdot 67^{\circ}$ v.a. (200 pixels) left and right from the center.

\subsubsection{Procedure}

The experiment started with a written instruction on the computer screen followed by a nine-point eye-tracking calibration and validation procedure. Only calibrations with an averaged accuracy below $1.0^{\circ}$ v.a. were accepted. The experiment consisted of 576 trials, separated in 6 blocks, plus one preceding practice trial. A feedback display after each block informed participants about the number of completed and total blocks. A trial began with a central colored object presented for 500 ms as target-object cue. Afterwards, participants had to keep fixation on a central plus (tolerance diameter of $2.5^{\circ} \mathrm{v} . \mathrm{a}$.) throughout a randomly chosen fixation period between $500 \mathrm{~ms}$ and 1,000 ms. Trials in which a participant did not manage to keep central fixation for the specified duration within an interval of 5 seconds were abandoned and repeated at a random position within the block. This time-out procedure was used to identify when the calibration was lost, e.g., due to a head movement. Calibration 
was repeated in this case. At the end of the fixation period, one colored object appeared left and another right from the center. The object with the same identity (shape) as the search cue was defined as the target, while the other object was a distractor. Participants were asked to saccade fast and accurate to the target object. The Experiment Builder software was used to detect saccade onsets online with a velocity threshold of $30 \%$ and an acceleration threshold of $8,000 \% \mathrm{~s}^{2}$. Trials in which participants did not start a saccade within $400 \mathrm{~ms}$ from search stimuli onset were abandoned and repeated at a random position within the block. A trial was completed by a fixation on the target (tolerance diameter of $2.8^{\circ} \mathrm{v} . \mathrm{a}$.) for $100 \mathrm{~ms}$ duration. A high-pitched tone informed participants about trial completion and the next trial was initiated. After the last trial, participants answered two post-experiment questions to reveal whether there had been any problems or misunderstandings.

\subsubsection{Design and analysis}

The experiment consisted of two conditions (Figure 1). In the no color-match condition, the cue, the target, and the distractor appeared in different colors. In the target color-match condition, the target color matched the cue color, while the distractor appeared in a different color. Both conditions were equally often completed per block in random order. Target and distractor location were also random, but matched per block and condition. Each color by object identity combination of the three objects $(24 \times 12=288)$ was used twice in random order across the experiment, whereby target color was substituted by the cue color in the color-match condition. Data were analyzed with Microsoft Excel 2010 and IBM SPSS Statistics 22. The dependent variables were proportion and median latency of the first saccades landing at the target (tolerance diameter of $2.8^{\circ} \mathrm{v}$.a. around the target). Paired $t$-tests were used to compare the dependent variables across the two conditions. A chance level of 
0.05 was applied as in all experiments. Data and analyses of all three experiments are provided as Supplementary Material (see data description at the end of this manuscript).

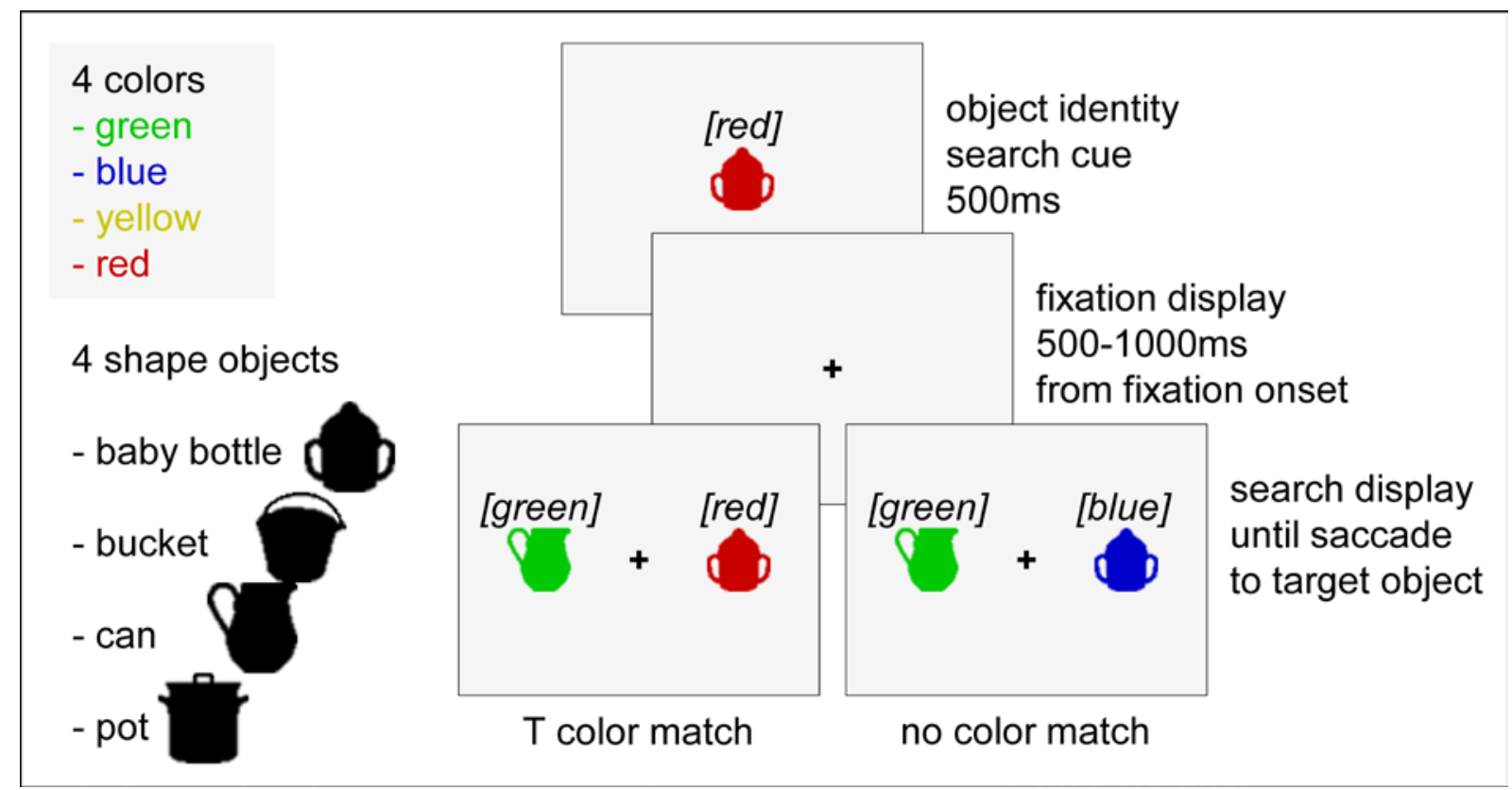

Figure 1. Material, procedure, and design of Experiment 1. The target was either presented in the same color as the search cue (T color match) or in a different color (no color match). The distractor was always presented in a different color than the search cue. The color words in squared brackets are added for greyscale printing and were not present during the experiment.

\subsection{Results}

On average two trials per participant had to be repeated because central fixation was not kept for the specified duration. On average twelve trials per participant had to be repeated because no saccade started within 400 ms from search stimuli onset. Three percent of all first saccades neither reached the target, nor the distractor (tolerance diameter of $2.8^{\circ} \mathrm{v}$.a.). The proportion was not different across the color match conditions $(t(7)=1.62, S E=.23, p=.15$, Cohen's $\left.d_{z}=.57\right)$. In terms of correct first saccades, $78 \%$ of the participants' first saccades landed at the target in case of the cue-target color match, (Figure 2, top). A significantly smaller proportion of first saccades, i.e., 59 \% reached the target region in the no color-match condition $\left(t(7)=8.95, S E=2.04, p<.0001\right.$, Cohen's $\left.d_{z}=3.16\right)$. In addition, the median latency of these target saccades was 9 ms shorter with than without a color match $(t(7)=6.45, S E=1.35$, $p<.001$, Cohen's $d_{z}=2.28$; Figure 2, bottom). Thus, saccades were faster and went more often 
to the target object, when cue and target matched in color than when no object matched the cue color.

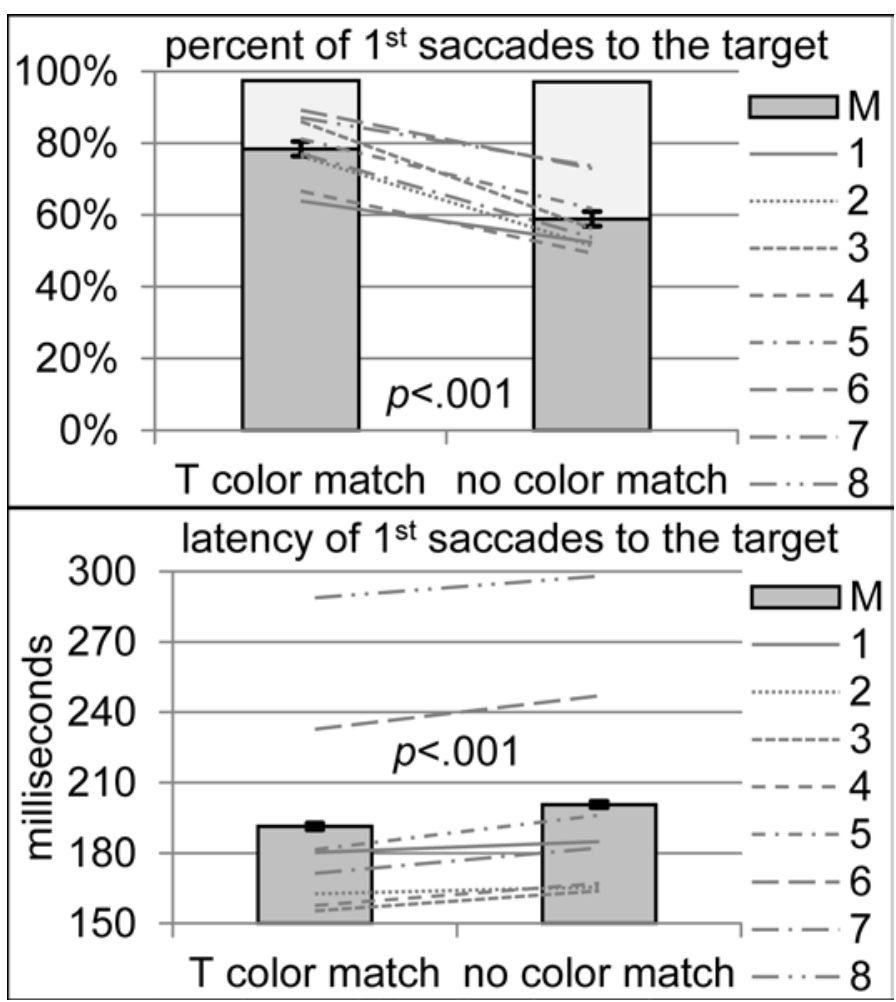

Figure 2. Results of Experiment 1. Percent (top) and median latency (bottom) of all first saccades reaching the target area in case of cue-target color match ( $\mathrm{T}$ color match) and in case of no color match between search cue and search objects (no color match). The lines represent individual subject data and the dark-grey bars represent sample means of the individual data. The light-grey bars in the upper diagram represent the percentage of first saccades reaching the distractor area. Error bars correspond to standard errors of the mean of the paired differences across color-match conditions.

\subsection{Discussion}

Although the identity of the cue defined the search target and its color was completely irrelevant, first saccades went more often towards the target if it appeared in the cue's color rather than any other color. The latency of the first saccade was also shorter in the colormatch condition. The results are in line with our hypothesis that trial-wise varying search targets are held as VWM templates in an object-based manner, so that all features including the irrelevant ones of a VWM template bias attentional selection.

However, from Experiment 1 it is not clear whether using a VWM template in an object-based manner to bias attention is a voluntary mechanism that is only applied if it is 
strategically useful. Because the distractor never appeared in the cue's color, cue color might have been voluntarily used as an additional search feature. In half of the trials, using cue color to bias saccadic selection was beneficial. In the remaining half, the cue color did not appear at all, so that a cue-color bias signal was also not detrimental. We reasoned therefore that if VWM templates work involuntarily in an object-based manner, then a searchirrelevant template feature should also bias attention towards a distractor having the same color as the cue. This possibility was investigated in a second experiment.

\section{Experiment 2}

As in Experiment 1, participants had to saccade to a target object defined by its identity and not by its color. To investigate whether a search-irrelevant VWM-template feature does even bias attention towards a distractor, not only the target, but also the distractor matched the cue's color in some trials. In addition, we added a condition in which both, target and distractor matched the search-irrelevant cue color. This condition was introduced to reveal whether faster saccadic responses are connected to the mere repetition of the cue's color, or whether it is necessary that the search objects can also be distinguished by color.

\subsection{Methods}

\subsubsection{Participants}

A new sample of eight students from Bielefeld University, Germany (3 males, 5 females; 29 years on average), participated in the experiment. All participants provided written informed consent prior to participation, were naïve with respect to the purpose of the study, reported normal or corrected-to-normal visual acuity, and were paid for participation. 


\subsubsection{Apparatus and stimuli}

Apparatus and stimuli were the same as in Experiment 1, except for a different set of real-world object shapes: a mug and a trophy (Figure 3). In addition, objects were only presented in the colors blue and red.

\subsubsection{Procedure}

The procedure was the same as in Experiment 1, except that the experiment consisted of 384 trials, separated in 4 blocks, plus one preceding practice trial.

\subsubsection{Design and analysis}

Experiment 2 consisted of four conditions (Figure 3). In the no color-match condition, the cue's color was different from the color of both, target and distractor, while target and distractor appeared in the same color. In the target color-match condition, the target color matched the cue's color, while the distractor appeared in the other color. In the distractor color-match condition, the distractor color matched the cue color, while the target appeared in the other color. In the both color-match condition, target and distractor appeared both in the cue color. All 32 combinations of conditions (4), locations (2), colors (2), and object identities (2) were repeated three times per block in random order. Thus, in contrast to Experiment 1, not only locations, but also colors and object identities were matched per block and condition. The same dependent variables were used as in Experiment 1. Within-subject analyses of variances were used to reveal whether the four conditions affected the dependent variables differently. Planned paired $t$-tests were used to compare the conditions to each other. 


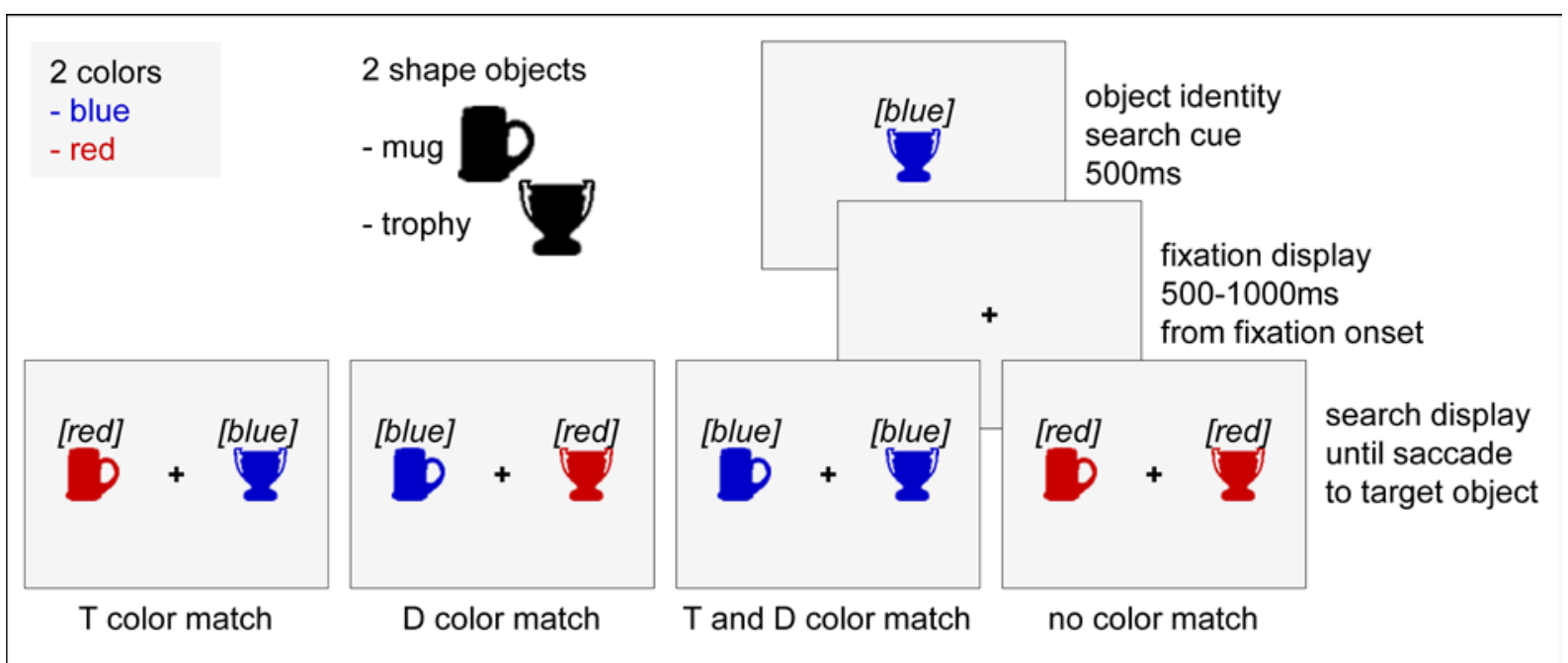

Figure 3. Material, procedure, and design of Experiment 2. Only the target (T color match), only the distractor (D color match), both ( $\mathrm{T}$ and $\mathrm{D}$ color match) or no search object (no color match) could be presented in the same color as the search cue. The color words in squared brackets are added for greyscale printing and were not present during the experiment.

\subsection{Results}

On average two trials per participant had to be repeated because central fixation was not kept for the specified duration. On average four trials per participant had to be repeated because no saccade began within 400 ms from search stimuli onset. Three percent of all first saccades neither reached the target, nor the distractor (tolerance diameter of $2.8^{\circ} \mathrm{v}$.a.). This proportion was not different across the four color-match conditions $(F(3,21)=.89, p=.47$, $\left.\eta_{p}{ }^{2}=.47\right)$. In terms of the correct first saccade, the proportion of first saccades landing directly at the target was significantly different across conditions $\left(F(3,21)=50.89, p<.001, \eta_{p}{ }^{2}=.88\right.$; Figure 4, top). More first saccades landed at the target in case of cue-target color match than without any color match between cue and search objects $(t(7)=5.95, S E=2.30, p<.001$, Cohen's $d_{z}=2.10$ ). This finding is a replication of the result in Experiment 1. Moreover, less first saccades went to the target in the distractor color-match condition than in the no colormatch condition $\left(t(7)=6.86, S E=3.55, p<.001\right.$, Cohen's $\left.d_{z}=2.43\right)$. The proportion of first saccades to the target was not significantly different across no color-match and both colormatch conditions $\left(t(7)=.59, S E=1.77, p=.58\right.$, Cohen's $\left.d_{z}=.21\right)$. The median latencies of the 
first target saccades differed significantly across color-match conditions $(F(3,21)=3.54$, $p<.05, \eta_{p}{ }^{2}=.34$; Figure 4 , bottom). Paired $t$-tests revealed that initial target saccades were significantly faster when only the target matched the cue in color than when no search object corresponded to the cue color $\left(t(7)=3.23, S E=4.68, p<.05\right.$, Cohen's $\left.d_{z}=1.14\right)$, replicating the finding of Experiment 1. In sum, first saccades went most often and fastest to the target, when only the target matched the cue color. First saccades went most often to the distractor if only the distractor matched the cue color. When target and distractor were colored the same, their correspondence to the cue color had no effect on saccade selection.

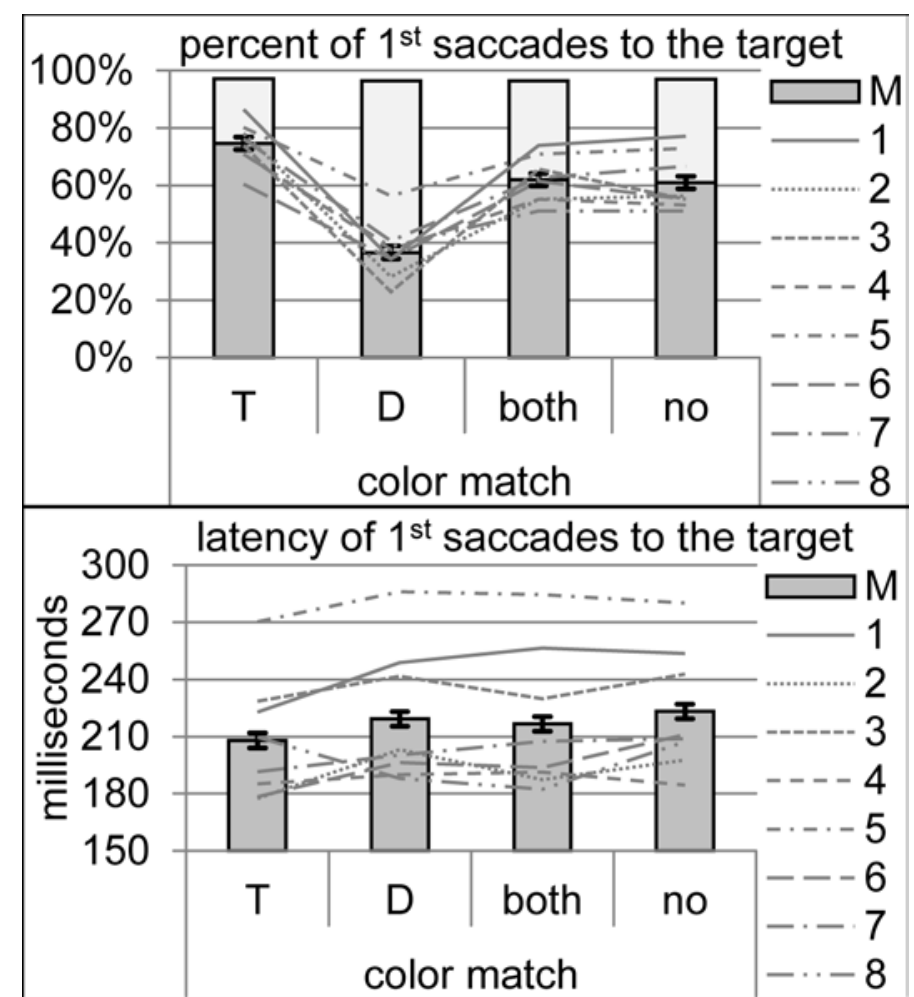

Figure 4. Results of Experiment 2. Percent (top) and median latency (bottom) of all first saccades reaching the target area in each of the four color-match conditions (T: only target matches cue color, D: only distractor matches cue color, both: target and distractor match cue color, none: neither target nor distractor match cue color). The lines represent individual subject data and the dark-grey bars represent sample means of the individual data. The lightgrey bars in the upper diagram represent the percentage of first saccades reaching the distractor area. Error bars correspond to standard errors of the means according to Loftus and Masson (1994), calculated as described in Franz and Loftus (2012). 


\subsection{Discussion}

As in Experiment 1, a cue-colored target led to more first target saccades than a different-colored target, replicating our key finding. Moreover, the cue-distractor color match was accompanied by fewer first saccades to the target and hence more first saccades to the distractor than when no search object corresponded to the cue color. Thus, oculomotor selection was biased likewise by cue-colored targets and distractors as long as color differentiated between the two. When target and distractor were of the same color, about 60 $\%$ of first saccades went to the target independent of whether both or no search object matched the cue in color. Thus, the effects are due to a selection bias towards cue-colored objects compared to different-colored objects. The effect is not based on faster reaction to cue-colored than different colored targets per se. Otherwise saccade latencies would have been faster in that condition, in which both search objects were of the same color as the search cue as compared to the condition without any color-match to the cue.

Still, participants might have used color voluntarily in Experiment 2. The attentional selection based on object identity alone was with about $60 \%$ in the no match-condition far from being perfect. In addition, color was valid in half of the trials and participants were not informed about the color-match probabilities prior to the experiment. Therefore, color might have been used voluntarily to increase selection efficiency in the cue-target color-matching trials. This possibility was investigated in a third experiment.

\section{Experiment 3}

In Experiments 1 and 2, participants might have voluntarily used color to bias attention allocation towards cue-colored items. Participants had not been informed about the color-match distributions in the previous two experiments and the target matched the cue's color in half of the trials. In addition, target selection according to object identity was a 
difficult task indicated by the performance level of about $60 \%$ correct first saccades in the no color-match baseline. Thus, biasing attention by color, although the target was defined by object identity was indeed beneficial in many trials and had the power to enhance target selection by about $20 \%$.

In the third experiment, we investigated whether a cue feature that hampers visual search captures attention as expected if VWM templates work in an object-based manner for attentional selection. Therefore, in the following experiment, the cue color never matched the target color, but matched the distractor color in half of the trials. This implies that the search object's color was uninformative in half of the trials, and even misleading in the remaining half. Furthermore, in order to increase search performance, peripheral discriminability of the search objects according to their identity was enhanced by using more dissimilar shaped objects. Finally, participants were informed that the color of the cue never matched the target, but matched the distractor in fifty percent of the trials.

\subsection{Methods}

\subsubsection{Participants}

A new sample of eight students from Bielefeld University, Germany (3 males, 5 females; 26 years on average) participated in the experiment after having provided written informed consent. Participants were naïve with respect to the purpose of the study, reported normal or corrected-to-normal visual acuity, and were paid for participation.

\subsubsection{Apparatus and stimuli}

Apparatus and stimuli were the same as in Experiment 1, except for a different set of real-world object shapes: a glass and a pot (Figure 5). The peripheral discriminability of these 
two objects is higher than the objects used so far. Therefore, finding the target according to its identity should be facilitated compared to the prior experiments.

\subsubsection{Procedure}

The procedure was the same as in Experiment 2, except that the written instruction informed participants that the target would never appear in the color of the search cue, while the distractor would appear in the cue color in fifty percent of all trials.

\subsubsection{Design and analysis}

Experiment 3 consisted of two conditions (Figure 5). In the no color-match condition, the target and the distractor appeared in different colors and neither of them matched the search cue's color. In the distractor-color match condition, the distractor appeared in the cue's color, while the target appeared in a different color. All combinations of conditions (2), locations (2), and object identities (2) were equally often completed per block in random order. Each of the 24 color combinations was used eight times in random order within each half of the experiment, whereby distractor color was substituted by the cue color in the colormatch condition. The dependent variables were the same as in the previous experiments. Two-sample t-tests were calculated to compare the no-color match baseline of this experiment to the two previous experiments. Paired $t$-tests were calculated to compare the dependent variables across the two conditions. 


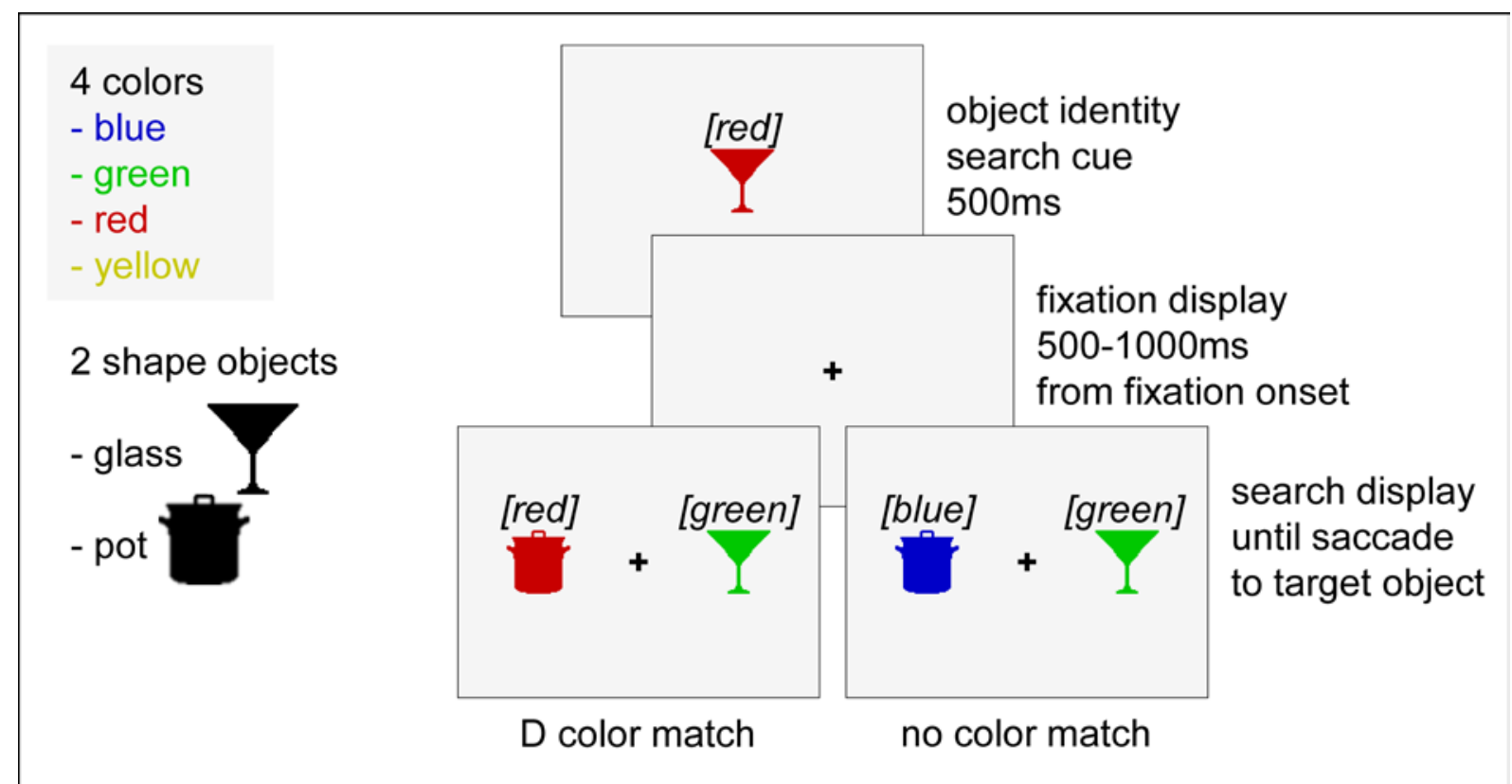

Figure 5. Material, procedure, and design of Experiment 3. The distractor was either presented in the same color as the search cue (D color match) or in a different color (no color match). The target was always presented in a different color than the search cue. The color words in squared brackets are added for greyscale printing and were not present during the experiment.

\subsection{Results}

On average three trials per participant had to be repeated because central fixation was not kept for the specified duration. On average eight trials per participant had to be repeated because no saccade began within $400 \mathrm{~ms}$ from search stimuli onset. Three percent of all first saccades neither reached the target, nor the distractor (tolerance diameter of $2.8^{\circ} \mathrm{v}$.a.). This proportion was not different across color-match conditions $(t(7)=1.17, S E=.34, p=.28$, Cohen's $\left.d_{z}=.41\right) .77 \%$ of the participants' first saccades landed directly at the target in the no color-match baseline (Figure 6, top). This is a significantly higher proportion as compared to the previous two experiments $\left(t(14)=3.08, S E=5.99, p<.01\right.$, Cohen's $d_{z}=1.65$ and $t(14)=2.72$, $S E=6.05, p<.05$, Cohen's $d_{z}=1.46$ ). In case of the cue-distractor color match, a significantly smaller proportion of saccades, i.e., $65 \%$ reached the target region, as compared to the no color match $\left(t(7)=6.32, S E=1.88, p<.001\right.$, Cohen's $\left.d_{z}=2.24\right)$, replicating and extending the finding of Experiment 2. Median latencies of the first target saccades were 4 ms slower in the 
distractor-match condition than in the no color-match condition $(t(7)=2.69, S E=0.86, p<.05$, Cohen's $d_{z}=.95$; Figure 6 , bottom). In sum, first saccades went less often and with a slower latency to the target object, when distractor and cue matched in color than when no object matched the cue color.

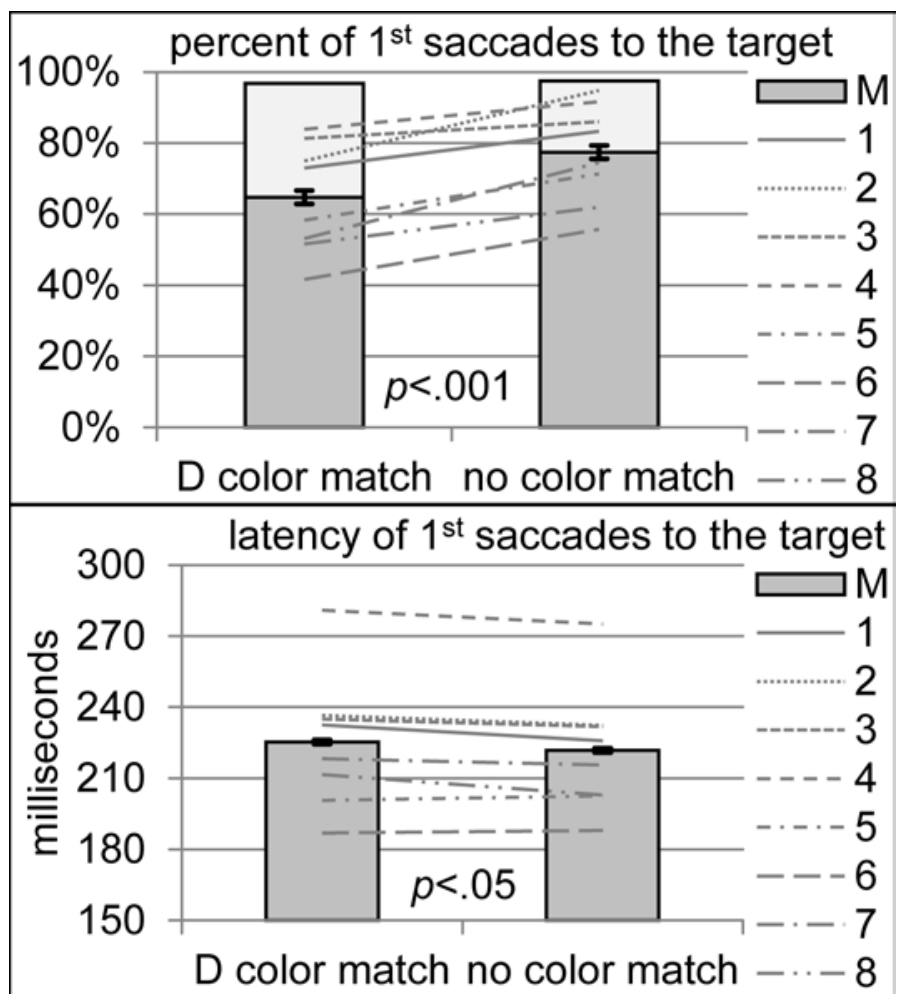

Figure 6. Results of Experiment 3. Percent (top) and median latency (bottom) of all first saccades reaching the target area in case of the cue-distractor color match (D color match) and in case of no color match between search cue and search objects (no color match). The lines represent individual subject data and the dark-grey bars represent sample means of the individual data. The light-grey bars in the upper diagram represent the percentage of first saccades reaching the distractor area. Error bars correspond to standard errors of the mean of the paired differences across color-match conditions.

\subsection{Discussion}

In Experiment 3, the color of the search cue was uninformative in half of the trials, and even misleading in the remaining half. Thus, it would have been strategically useful to downweigh any cue-colored object or even to ignore color completely in Experiment 3. Nevertheless, initial saccades landed still more frequently on a cue-colored than on a different-colored distractor. This was the case, although the search objects could be better discriminated peripherally on the basis of their shape as compared to the previous 
experiments. The results of Experiment 3 further support the hypothesis that a VWM-search template works in an object-based and involuntary manner. All features of a VWM template seem to bias attentional and saccadic selection obligatorily by an involuntary top-down regulation. Task knowledge cannot be used to eliminate this form of oculomotor capture by search-irrelevant template-matching features.

\section{General Discussion}

When a search task requires changing the search target from one search to the next, humans seem to rely on VWM to bias attention towards template-matching objects in the search region (Conci, Müller, \& von Mühlenen, 2013; Hollingworth, 2012; Wolfe, 1994; Woodman \& Chun, 2006). While top-down bias signals are configured to favor task-relevant features (Bundesen, 1990; Desimone \& Duncan, 1995; Foerster \& Schneider, 2015), whole objects in terms of bound features seem to be retained in VWM (Luck \& Vogel, 1997; Vogel et al., 2001). Therefore, the question arises whether VWM templates for visual search bias attentional selection by task-relevant features only, or whether the bias refers to all features of the search template. To answer this question, we investigated whether the search-irrelevant color of a VWM template biases selection in a visual search task with trial-wise changing targets. In three experiments, participants had to saccade to a target object according to its identity and irrespective of its color. Although object identity and not color defined the target, initial saccades went more often and faster to targets that matched the search cue in color than to different-colored targets in Experiment 1. This result indicates that both features of the VWM template created an attentional bias signal in an object-based manner. In Experiment 2, attentional selection was likewise biased towards cue-colored targets and, importantly, also towards distractors, indicating that the search-irrelevant color of the VWM template was also applied if it was occasionally invalid. In Experiment 3, when the color of the search cue was 
never valid and even misleading in half of the trials, cue-colored distractors still captured the eyes more frequently than different-colored distractors. This was the case, although participants were informed about the misleading nature of the cue color. This result indicates that task knowledge could not be used to strategically downweigh cue-colored objects or to ignore color information completely. Instead, in the VWM-based search attentional biasing seemed to be based also on the irrelevant color features of the search target, and this biasing was obligatory. We conclude that VWM templates bias attention involuntarily in an objectbased manner.

The reported results are related to a different line of research investigating attentional biasing effects of VWM contents in dual task studies (Gao et al., 2016; Olivers, Meijer, \& Theeuwes, 2006; Sala \& Courtney, 2009; Soto \& Humphreys, 2009). In this line of research, a first task requires to store items in VWM for later use. During the retention phase, it is investigated how the VWM content biases attention in a second visual-search task. Results show that VWM content which is completely irrelevant for the ongoing search task can bias attention towards VWM-matching items (Downing, 2000; Olivers, 2009; Olivers et al., 2006; Soto et al., 2005; Soto, Hodsoll, Rotshtein, \& Humphreys, 2008; Soto et al., 2006; Soto \& Humphreys, 2009). For instance, in the experiment series of Soto and colleagues, participants first encoded a colored shape for a later same-different judgment task. While maintaining the colored shape in VWM, they had to search for a tilted line in a visual display. Participants found the tilted line fastest, when it was surrounded by a VWM-matching item and slowest when a VWM-matching item was present that contained a distractor line. Thus, attention was biased towards VWM-matching items during the visual search, although the VWM content was completely irrelevant for the search task. This finding was interpreted as evidence for involuntary top-down control by VWM content. However, attentional biasing by VWM content during interim search tasks is not always observed (Downing \& Dodds, 2004; Han \& 
Kim, 2009; Olivers et al., 2006; Sala \& Courtney, 2009; Woodman \& Luck, 2007). In some investigations, a VWM-matching distractor could even be more efficiently ignored than distractors that did not match the VWM content (Han \& Kim, 2009; Woodman \& Luck, 2007). How can the conflicting results be explained?

Olivers et al. (2011) suggested that in order to bias attentional selection towards VWM-matching material, VWM representations must be in an active rather than in an accessory state. The idea is that only one item at a time can be in an active VWM state and that this item has an automatic influence on attentional selection. Simultaneously, VWM can maintain further items in an accessory state. These items can be remembered later, but do not or only slightly influence ongoing attentional selection. Which item will be kept in an active state is determined by task requirements. On a neuronal basis, items maintained in the two different VWM states might be coded by different neuronal populations. Only the neurons coding active VWM items may provide feedback to lower visual areas (Sigala, Kusunoki, Nimmo-Smith, Gaffan, \& Duncan, 2008; Warden \& Miller, 2007, 2010), thereby interacting with selective attention. Related to this idea, the task-driven theory of visual attention and working memory (TRAM, Schneider, 2013) distinguishes between activation-based and passive VWM maintenance. Activation-based VWM maintenance is accomplished via reverberating neuronal loops involving brain areas coding visual features (Bundesen et al., 2005; Bundesen, Habekost, \& Kyllingsbæk, 2011). Passive VWM maintenance might be accomplished via short-term synaptic changes (cf. Stokes et al., 2013) after sufficient looping duration in the activation-based state. The transfer from active to passive VWM guarantees precisely that visual representations can be maintained without much interference with ongoing attentional biasing (e.g., in interim tasks or across saccades, cf. Foerster, Carbone, \& Schneider, 2014; Poth, Herwig, \& Schneider, 2015; Poth \& Schneider, 2016). While the conceptions of the accessory and the passive state differ in some aspects (see Olivers et al., 
2011; Schneider, 2013), the conceptions of the active and activation-based state agree on the attentional biasing effect. In addition, a VWM representation leaves the active state and is transferred into an accessory or passive state if it is irrelevant for the ongoing task.

When having to keep something in VWM for later recognition during interim visual searches, as was the case in the reported dual-task studies, it is useful to keep the VWM content in an accessory or passive state in order to prevent interference. Olivers et al. (2011) argued that the VWM item might have been kept in an active state in some experiments due to strategical reasons. Often, attending the VWM-matching item in the search display was not detrimental or even supportive for the visual search, e.g., in case of being over-proportionally valid (Soto et al., 2005, 2008, 2006). Attending VWM-matching items (in an active state) during search is also a sensible rehearsal strategy to improve later recall. Finally, short cuepresentation times may have prevented transformation into an accessory state prior to the onset of the search display (cf. argumentation in Han \& Kim, 2009 and Olivers et al., 2011).

Importantly, in contrast to the reported previous dual-task studies on VWM and attentional biasing, we investigated here in a single VWM-based search task how the searchirrelevant color of the search target influenced attentional selection. The VWM object which constituted the search target was relevant for the ongoing search and thus needed to be kept in an active state as an attentional VWM template. A central open question was whether all the features of this active VWM template bias attention in an object-based manner, or whether only the search-relevant shape biases attention. Our results revealed that the search-irrelevant color of the search cue biased attention even if this was detrimental for the ongoing search task. Based on these results, we argue that all active features of a VWM search template bias attentional selection by an involuntary and object-based top-down mechanism. This finding is also in line with neuronal evidence for object-based attentional selection (O'Craven, Downing, \& Kanwisher, 1999; Roelfsema, Lamme, \& Spekreijse, 1998). 
In the current study, we always used color as irrelevant and shape as relevant search dimension. Therefore, the question arises whether a similar pattern would be found with other feature combinations. Only if this is the case, our theoretical conclusions are generalizable beyond the feature color. Fortunately, the dual-task line of research on VWM and attention can provide first insights into this question. Among these dual-task studies on VWM and attention, a few studies asked whether all or only relevant features of a VWM item bias attention (Gao et al., 2016; Olivers et al., 2006; Sala \& Courtney, 2009; Soto \& Humphreys, 2009). Participants in Gao et al. (2016) were asked to memorize the color of a colored shape for later recall. During the retention interval, they had to search for a tilted target line among vertical distractor lines. All lines were surrounded by colored shapes. The shapes surrounding the distractor lines could match the memory item in color, shape, both, or no feature. Search times were prolonged in all match conditions compared to the no match condition, arguing that in addition to the memory-task-relevant color also the memory-task-irrelevant shape information was retained in VWM and biased attention during the interim search task. Importantly, this result shows that object-based biasing can also be found with shape as the irrelevant object dimension. This finding strengthens our previous conclusions of involuntary object-based attentional biasing, assuming that similar mechanisms are responsible for the dual-task and the single-task biasing effects. In addition, instead of real-word objects, Gao et al. (2016) as well as Soto and Humphreys (2009) used geometric forms like triangles, circles, and squares. In Soto and Humphreys (2009), the color of the to-be-remembered geometric forms was irrelevant for a later match-to-sample task and nevertheless biased attention during an interim search task, indicated by prolonged search times.

However, object-based biasing was not found in all dual-task studies (Olivers et al., 2006; Sala \& Courtney, 2009). In Experiment 4 of Olivers et al. (2006), participants had to remember either the shape or the color of a colored shape for later recognition. In-between 
encoding and recognition, participants searched for a size singleton in an array of circles and responded with a two-choice button press to its containing letter (M vs. N). Besides the target singleton, a distractor singleton was presented among the search objects. A singleton distractor in a singleton-search task is known to slow search times (Horstmann, Becker, \& Ernst, 2016; Lamy \& Egeth, 2003; Theeuwes \& Burger, 1998). Severe slowing was observed by Olivers et al. (2006) when the singleton distractor resembled the relevant feature of the memory item (shape similarity in shape memory task and color similarity in color memory task), but no slowing was observed when the singleton distractor resembled the irrelevant feature of the to-be-memorized item (color similarity in shape memory task and shape similarity in color memory task). Also Sala and Courtney (2009) found task-dependent biasing when participants had to remember either the texture or the location of memory items. The authors concluded that VWM can selectively prioritize task-relevant features. How are these findings compatible with our assumption that VWM-based search works via object-based attentional templates? Object-based biasing does not imply that all features whether task-relevant or not - should exert the same biasing strength. Task-relevant features of the search object should have a stronger biasing effect (Bundesen, 1990). Importantly, as shown in our three experiments, in the visual search situations with changing search targets, the search-irrelevant color feature was not only retained in VWM but it exerted also a bias effect in the search process. Presumably, the biasing strength of a VWM feature depends not only on its task-relevance but also on the specific feature and its characteristics. As color was always the irrelevant feature in our experiments, future research has to clarify whether similar results can be obtained with other feature combinations in our single-task paradigm just like it has been found in the dual-task paradigm. 


\section{Acknowledgement}

This research was supported by the Cluster of Excellence Cognitive Interaction Technology 'CITEC' (EXC 277) at Bielefeld University, which is funded by the German Research Foundation (DFG).

\section{Supplementary Material}

Supplementary data files can be found on Cognition's website at https://doi.org/10.1016/j.cognition.2017.12.002. The data of all three experiments as well as the Excel calculations are provided within one Microsoft Excel file as Supplementary Material. The file contains seven Excel charts. The first Excel chart named "Exp1data" contains the data of Experiment 1. The second Excel chart named "Exp1ana” contains all Excel analysis calculated based on the data of Experiment 1 as well as the data plots. The third Excel chart named "Exp2data" contains the data of Experiment 2. The forth Excel chart named "Exp2ana” contains all Excel analysis calculated based on the data of Experiment 2, the paired difference tables imported from SPSS for the calculation of the standard errors of the means based on the method described in Franz and Loftus (2012) as well as the data plots. The fifth Excel chart named "Exp3data" contains the data of Experiment 3. The sixth Excel chart named "Exp3ana" contains all Excel analysis calculated based on the data of Experiment 3 as well as the data plots. The seventh Excel chart named "labels" contains the labels for all column names and a description of their content as well as the selection options for answering the last post-experiment question.

\section{References}

Alvarez, G. A., \& Cavanagh, P. (2004). The capacity of visual short-term memory is set both by visual information load and by number of objects. Psychological Science, 15, 106- 
111. doi:10.1111/j.0963-7214.2004.01502006.x

Baddeley, A. D. (1986). Working Memory. Oxford University Press.

Baddeley, A. D., \& Hitch, G. (1974). Working memory.

Bravo, M. J., \& Nakayama, K. (1992). The role of attention in different visual-search tasks. Perception and Psychophysics, 51, 465-472. doi:10.3758/BF03211642

Bundesen, C. (1990). A theory of visual attention. Psychological Review, 97, 523-547.

Bundesen, C., Habekost, T., \& Kyllingsbaek, S. (2005). A neural theory of visual attention: Bridging cognition and neurophysiology. Psychological Review, 112, 291-328. doi:10.1037/0033-295X.112.2.291

Bundesen, C., Habekost, T., \& Kyllingsbæk, S. (2011). A neural theory of visual attention and short-term memory (NTVA). Neuropsychologia, 49, 1446-1457. doi:10.1016/j.neuropsychologia.2010.12.006

Conci, M., Müller, H. J., \& von Mühlenen, A. (2013). Object-based implicit learning in visual search: Perceptual segmentation constrains contextual cueing. Journal of Vision, 13(3), 1-17. doi:10.1167/13.3.15.doi

Cowan, N. (2000). The magical number 4 in short-term memory: A reconsideration of mental storage capacity Dep. Behavioral and Brain Sciences, 24, 87-185.

D’Esposito, M., \& Postle, B. R. (2015). The Cognitive Neuroscience of Working Memory. Annual Review of Psychology, 66, 115-142. doi:10.1146/annurev-psych-010814-015031

Desimone, R., \& Duncan, J. S. (1995). Neural mechanisms of selective visual attention. Annual Review of Neuroscience, 18, 193-222. doi:10.1146/annurev.ne.18.030195.001205

Deubel, H., \& Schneider, W. X. (1996). Saccade target selection and object recognition: 
Evidence for a common attentional mechanism. Vision Research, 36, 1827-1837. doi:10.1016/0042-6989(95)00294-4

Downing, P. E. (2000). Interactions between visual working memory and selective attention. Psychological Science, 11, 467-473. doi:10.1111/1467-9280.00290

Downing, P. E., \& Dodds, C. M. (2004). Competition in visual working memory for control of search. Visual Cognition, 11, 689-703. doi:10.1080/13506280344000446

Foerster, R. M., Carbone, E., \& Schneider, W. X. (2014). Long-term memory-based control of attention in multi-step tasks requires working memory: Evidence from domainspecific interference. Frontiers in Psychology, 5, 1-8. doi:10.3389/fpsyg.2014.00408

Foerster, R. M., \& Schneider, W. X. (2015). Expectation violations in sensorimotor sequences: shifting from LTM-based attentional selection to visual search. Annals of the New York Academy of Sciences, 1339, 45-59. doi:10.1111/nyas.12729

Franz, V. H., \& Loftus, G. R. (2012). Standard errors and confidence intervals in withinsubjects designs: Generalizing Loftus and Masson (1994) and avoiding the biases of alternative accounts. Psychonomic Bulletin and Review, 19, 395-404. doi:10.3758/s13423-012-0230-1

Fukuda, K., Awh, E., \& Vogel, E. K. (2010). Discrete capacity limits in visual working memory. Current Opinion in Neurobiology, 20, 177-182. doi:10.1016/j.conb.2010.03.005.Discrete

Gao, Z., Yu, S., Zhu, C., Shui, R., Weng, X., Li, P., \& Shen, M. (2016). Object-based encoding in visual working memory: Evidence from memory-driven attentional capture. Scientific Reports, 6, 22822. doi:10.1038/srep22822

Han, S. W., \& Kim, M.-S. (2009). Do the contents of working memory capture attention? 
Yes, but cognitive control matters. Journal of Experimental Psychology: Human Perception and Performance, 35, 1292-1302. doi:10.1037/a0016452

Hardman, K. O., \& Cowan, N. (2015). Remembering complex objects in visual working memory: do capacity limits restrict objects or features? J Exp Psychol Learn Mem Cogn, 41, 325-347. doi:10.1037/xlm0000031

Hollingworth, A. (2012). Guidance of visual search by memory and knowledge. In M. D. Dodd \& J. H. Flowers (Eds.), The influence of attention, learning, and motivation on visual search (pp. 63-89). New York, NY: Springer New York. doi:10.1007/978-1$4614-4794-8$

Horstmann, G., Becker, S., \& Ernst, D. (2016). Perceptual salience captures the eyes on a surprise trial. Attention, Perception, and Psychophysics, 78, 1889-1900. doi:10.3758/s13414-016-1102-y

Kahneman, D., Treisman, A., \& Gibbs, B. J. (1992). The reviewing of object files: Objectspecific integration of information. Cognitive Psychology, 24, 175-219.

Konkle, T., Brady, T. F., Alvarez, G. A., \& Oliva, A. (2010). Conceptual distinctiveness supports detailed visual long-term memory for real-world objects. Journal of Experimental Psychology: General, 139, 558-578. doi:10.1037/a0019165

Lamy, D., \& Egeth, H. E. (2003). Attentional capture in singleton-detection and featuresearch modes. Journal of Experimental Psychology: Human Perception and Performance, 29, 1003-1020. doi:10.1037/0096-1523.29.5.1003

Loftus, G. R., \& Masson, M. E. J. (1994). Using confidence intervals in within-subject designs. Psychonomic Bulletin and Review, 1, 476-490. doi:10.3758/BF03210951

Luck, S. J., \& Vogel, E. K. (1997). The capacity of visual working memory for features and 
conjunctions. Nature, 390, 279-81. doi:10.1038/36846

Luck, S. J., \& Vogel, E. K. (2013). Visual working memory capacity: from psychophysics and neurobiology to individual differences. Trends in Cognitive Sciences, 17, 391-400. doi:10.1016/j.tics.2013.06.006

Ma, W. J., Husain, M., \& Bays, P. M. (2014). Changing concepts of working memory. Nature Neuroscience, 17, 347-356. doi:10.1038/nn.3655

O’Craven, K. M., Downing, P. E., \& Kanwisher, N. (1999). fMRI evidence for objects as the units of attentional selection. Nature, 401, 584-587. doi:10.1038/44134

Oberauer, K. (2009). Design for a working memory. In B. H. Ross (Ed.), Advances in research and theory (Vol. 51, pp. 45-100). San Diego: Elsevier Academic. doi:10.1016/S0079-7421(09)51002-X

Olivers, C. N. L. (2009). What drives memory-driven attentional capture? The effects of memory type, display type, and search type. Journal of Experimental Psychology: Human Perception and Performance, 35, 1275-1291. doi:10.1037/a0013896

Olivers, C. N. L., Meijer, F., \& Theeuwes, J. (2006). Feature-based memory-driven attentional capture: Visual working memory content affects visual attention. Journal of Experimental Psychology: Human Perception and Performance, 32, 1243-1265. doi:10.1037/0096-1523.32.5.1243

Olivers, C. N. L., Peters, J., Houtkamp, R., \& Roelfsema, P. R. (2011). Different states in visual working memory: when it guides attention and when it does not. Trends in Cognitive Sciences, 15, 327-334. doi:10.1016/j.tics.2011.05.004

Palmer, J., Boston, B., \& Moore, C. M. (2015). Limited capacity for memory tasks with multiple features within a single object. Attention, Perception, \& Psychophysics, 77, 
1488-1499. doi:10.3758/s13414-015-0909-2

Poth, C. H., Herwig, A., \& Schneider, W. X. (2015). Breaking object correspondence across saccadic eye movements deteriorates object recognition. Frontiers in Systems Neuroscience, 9, 1-10. doi:10.3389/fnsys.2015.00176

Poth, C. H., \& Schneider, W. X. (2016). Breaking object correspondence across saccades impairs object recognition: The role of color and luminance. Journal of Vision, 16(11):1, 1-12. doi:10.1167/16.11.1.doi

Roelfsema, P. R., Lamme, V. A. F., \& Spekreijse, H. (1998). Object-based attention in the primary visual cortex of the macaque monkey. Nature, 395, 376-381. doi:10.1038/26475

Rossi, A. F., Harris, B. J., Bichot, N. P., Desimone, R., \& Ungerleider, L. G. (2001). Deficits in target selection in monkeys with prefrontal lesions. Society for Neuroscience Abstracts, 574-579.

Sala, J. B., \& Courtney, S. M. (2009). Flexible working memory representation of the relationship between an object and its location as revealed by interactions with attention. Attention, Perception and Psychophysics, 71, 1525-1533. doi:10.3758/APP.71.7.1525

Schneider, W. X. (1995). VAM: A neuro-cognitive model for visual attention control of segmentation, object recognition, and space-based motor action. Visual Cognition, 2, 331-376. doi:10.1080/13506289508401737

Schneider, W. X. (2013). Selective visual processing across competition episodes: a theory of task-driven visual attention and working memory. Philosophical Transactions of the Royal Society B: Biological Sciences, 368, 1-13. doi:10.1098/rstb.2013.0060

Sigala, N., Kusunoki, M., Nimmo-Smith, I., Gaffan, D., \& Duncan, J. (2008). Hierarchical 
coding for sequential task events in the monkey prefrontal cortex. Proceedings of the National Academy of Sciences of the United States of America, 105, 11969-11974. doi:10.1073/pnas.0802569105

Soto, D., Heinke, D., Humphreys, G. W., \& Blanco, M. J. (2005). Early, involuntary topdown guidance of attention from working memory. Journal of Experimental Psychology: Human Perception and Performance, 31, 248-261. doi:10.1037/00961523.31.2.248

Soto, D., Hodsoll, J., Rotshtein, P., \& Humphreys, G. W. (2008). Automatic guidance of attention from working memory. Trends in Cognitive Sciences, 12, 342-348. doi:10.1016/j.tics.2008.05.007

Soto, D., \& Humphreys, G. W. (2009). Automatic selection of irrelevant object features through working memory. Experimental Psychology, 56, 165-172. doi:10.1027/16183169.56.3.165

Soto, D., Humphreys, G. W., \& Heinke, D. (2006). Working memory can guide pop-out search. Vision Research, 46, 1010-1018. doi:10.1016/j.visres.2005.09.008

Stokes, M. G., Kusunoki, M., Sigala, N., Nili, H., Gaffan, D., \& Duncan, J. (2013). Dynamic coding for cognitive control in prefrontal cortex. Neuron, 1-12. doi:10.1016/j.neuron.2013.01.039

Theeuwes, J., \& Burger, R. (1998). Attentional control during visual search: The effect of irrelevant singletons. Journal of Experimental Psychology: Human Perception and Performance, 24, 1342-1353. doi:10.1037/0096-1523.24.5.1342

Treisman, A. M., \& Gelade, G. (1980). A feature-integration theory of attention. Cognitive Psychology, 12, 97-136. doi:10.1016/0010-0285(80)90005-5 
Vogel, E. K., \& Machizawa, M. G. (2004). Neural activity predicts individual differences in visual working memory capacity. Nature, 428, 748-751. doi:10.1038/nature02447

Vogel, E. K., Woodman, G. F., \& Luck, S. J. (2001). Storage of features, conjunctions, and objects in visual working memory. Journal of Experimental Psychology: Human Perception and Performance, 27, 92-114. doi:10.1037//0096-1523.27.1.92

Warden, M. R., \& Miller, E. K. (2007). The Representation of Multiple Objects in Prefrontal Neuronal Delay Activity. Cerebral Cortex, 17, i41-i50. doi:10.1093/cercor/bhm070

Warden, M. R., \& Miller, E. K. (2010). Task-Dependent Changes in Short-Term Memory in the Prefrontal Cortex. Journal of Neuroscience, 30, 15801-15810. doi:10.1523/JNEUROSCI.1569-10.2010

Wolfe, J. M. (1994). Guided Search 2.0: A revised model of visual search. Psychonomic Bulletin and Review, 1, 202-238. doi:10.3758/BF03200774

Wolfe, J. M. (2010). Visual search. Current Biology, 20, R346-R349. doi:10.1016/j.cub.2010.02.016

Wolfe, J. M., Horowitz, T. S., Kenner, N., Hyle, M., \& Vasan, N. (2004). How fast can you change your mind? The speed of top-down guidance in visual search. Vision Research, 44, 1411-1426. doi:10.1016/j.visres.2003.11.024

Woodman, G. F., Carlisle, N. B., \& Reinhart, R. M. G. (2013). Where do we store the memory representations that guide attention? Journal of Vision, 13(3), 1-17. doi:10.1167/13.3.1.doi

Woodman, G. F., \& Chun, M. M. (2006). The role of working memory and long-term memory in visual search. Visual Cognition, 14, 808-830. doi:10.1080/13506280500197397 
Woodman, G. F., \& Luck, S. J. (2007). Do the contents of visual working memory automatically influence attentional selection during visual search? Journal of Experimental Psychology: Human Perception and Performance, 33, 363-377. doi:10.1037/0096-1523.33.2.363

Zelinsky, G. J. (2012). TAM: Explaining off-object fixations and central fixation tendencies as effects of population averaging during search. Visual Cognition, 20, 515-545. doi:10.1080/13506285.2012.666577 